\title{
Syphilis in Ottawa: An evolving epidemic
}

\author{
Lauren Orser ${ }^{1,2 \star}$, Paul MacPherson ${ }^{3,4,5}$, Patrick O’Byrne ${ }^{1,2}$
}

\begin{abstract}
Background: The incidence of infectious syphilis in Canada has declined throughout the latter decades of the last century; however, in Ottawa, an upsurge in new cases began in 2001. The local epidemic continues to involve predominantly gay, bisexual and other men who have sex with men (gbMSM), but in recent years, has expanded further into heterosexual populations. This has coincided with an increase in the number of pregnant women testing positive for syphilis on antenatal screening. The aim of this study is to understand the changing epidemiology in infectious syphilis cases diagnosed in Ottawa to strengthen primary care management and public health response.
\end{abstract}

Methods: Surveillance data from the Ontario Ministry of Health were used to describe the evolving epidemiology of infectious syphilis in the Ottawa region from 2010 to 2019, including a comprehensive chart review of cases from 2015-2019.

Results: The number of cases of infectious syphilis in Ottawa rose from 50 cases in 2010 to 171 cases in 2019. These rates were consistently high among males, and increased from $10.9 / 100,000$ in 2010 to $30.9 / 100,000$ in 2019. The rates among females, in comparison, increased from $0.4 / 100,000$ in 2010 to $3.2 / 100,000$ in 2019, with corresponding increases during antenatal screening (with no congenital syphilis cases to date).

Conclusion: As the syphilis epidemic continues to evolve in Ottawa, ongoing surveillance plays a crucial role. Public health resources must address the needs of populations already impacted but at the same time be flexible enough to respond to changes in trends and support clinicians providing care to populations where the epidemic is emerging.
This work is licensed under a Creative Commons Attribution 4.0 International License.

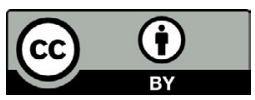

Affiliations

${ }^{1}$ School of Nursing, Faculty of Health Sciences, University of Ottawa, Ottawa, ON

2 Ottawa Public Health, Sexual Health Services Unit, Ottawa, ON

${ }^{3}$ Division of Infectious Diseases, The Ottawa Hospital, Ottawa, ON

${ }^{4}$ Clinical Epidemiology Program, The Ottawa Hospital Research Institute, Ottawa, ON

${ }^{5}$ Department of Medicine, Faculty of Medicine, University of Ottawa, Ottawa, ON

\section{*Correspondence:}

lorse060@uottawa.ca

Suggested citation: Orser L, MacPherson P, O'Byrne P. Syphilis in Ottawa: An evolving epidemic.

Can Commun Dis Rep 2022;48(2/3):76-82. https://doi.org/10.14745/ccdr.v48i23a04

Keywords: syphilis, syphilis priority groups, antenatal syphilis, surveillance data

\section{Introduction}

Across Canada, there has been an upsurgence in infectious syphilis. While cases reached an all time low through the 1990s, the incidence of infectious syphilis began to increase in 2001 with most diagnoses occurring among gay, bisexual and other men who have sex with men (gbMSM) (1). Since 2010, however, there has been a secondary surge in the incidence of infectious syphilis with an increasing number of cases now being reported among persons previously thought to be at low risk including heterosexual men and, in particular, women of childbearing age $(2,3)$. Indeed, over just a three-year period from 2015 to 2018, the rate of infectious syphilis among Canadian women aged 20 to 24 years increased nearly eight-fold from 3.4 to 26.0 per 100,000 (4). Such reports have coincided with an increase in the number of pregnant women testing positive for syphilis on antenatal screening and a 1,725\% increase in cases of congenital syphilis in Canada (5).
In light of the changing landscape of syphilis in Canada, we sought to re-evaluate what is known about syphilis epidemiology in Ottawa. We undertook a retrospective review of syphilis diagnoses from 2010 to 2019 with a more comprehensive analysis of persons diagnosed with infectious syphilis from 2015-2019. The objective of this review was to identify trends in syphilis epidemiology and case characteristics to better inform prevention and management efforts.

\section{Background}

While reports of syphilis infection were historically noted in Ottawa, these diagnoses were rare (fewer than $0.5 / 100,000$ ) and most were of late latent infection (6). In 2001, however, Ottawa Public Health (OPH) reported four cases of early infectious syphilis (rate: $0.5 / 100,000)(6)$, the first sign of new transmission 
within the region. By 2006, the number of new cases had increased 10-fold to 41 (rate: 4.8/100,000) (6). A review of cases from January 2001 to June 2006 (7), revealed a predominantly male epidemic with a male-to-female ratio of 19:1, with gbMSM accounting for the majority of cases (83.5\%). Nearly half (43\%) of those diagnosed with infectious syphilis were human immunodeficiency virus (HIV)-positive (7). Oral-genital contact was the most predominant form of transmission and nearly half of epidemiologically linked sexual partners resided outside Ottawa (7). Sexual networks involving Montréal and Toronto were the most common. Since 2006, the rate of new syphilis infections across Ontario has continued to rise with a $242 \%$ increase from 2010 to 2019 (8), and, like elsewhere in Canada (4), heterosexual men and women now make up an increasing proportion of new cases. In view of this, we sought to understand the changing epidemiology in Ottawa. Ongoing surveillance will play a crucial role in guiding clinicians and public health officials and informing screening and prevention efforts.

\section{Methods}

This review occurred in Ottawa, Canada, a city with a population of over one million based on 2019 data. The main objectives of this review were to 1 ) obtain a comprehensive picture of syphilis epidemiology in Ottawa and 2) improve our understanding of the characteristics and risk factors for infection among persons diagnosed with syphilis. To achieve these aims, we completed a review of all syphilis cases diagnosed in Ottawa from 2010 to 2019, focusing on local syphilis epidemiology. We also completed an in-depth chart review of infectious syphilis cases from 2015 (following a major increase in syphilis rates) to 2019 (the most recent available case data) and a review of cases diagnosed during pregnancy from 2015 to 2021 (using preliminary data from 2020-2021).

For the overall epidemiologic review (2010-2019), cases were classified as either infectious (primary, secondary and early latent) or late latent. Data were obtained using the following: 1) the Public Health Ontario (PHO) reportable diseases report, which provides counts of infectious syphilis diagnoses from $\mathrm{OPH} ; 2$ ) the City of Ottawa infectious diseases reports, which tabulate infectious and non-infectious syphilis diagnoses locally; and 3) the Integrated Public Health Information System database, which contains information about cases diagnosed within the province, including names, demographic, diagnosis and treatment information. Duplicate cases were removed. Case counts were logged in an Excel spreadsheet by year based on diagnosis (infectious or late latent syphilis).

A more in-depth analysis was conducted by chart review of public health charts (obtained from OPH) for cases of infectious syphilis diagnosed from 2015 to 2019 . We considered only diagnoses of primary, secondary and early latent syphilis among persons who resided in Ottawa during this period. Cases were excluded if they were diagnosed as syphilis of unknown duration, late latent syphilis, or neurosyphilis. In addition, syphilis contacts (i.e. those with a known exposure but not diagnosed with syphilis) were also excluded. Of a total 672 cases of infectious syphilis in Ottawa identified using the above datasets, 637 charts (95\% of the total cases) were available for review. The 35 charts not available for review related to a discrepancy in the number of infectious syphilis cases reported by $\mathrm{PHO}$ (672 cases) compared to the number retrieved during the chart review (637 cases). For data collection, we extracted age, gender (as reported), sexual orientation, history of other sexually transmitted infections, HIV status at time of diagnosis and pregnancy status (where applicable). All data were recorded in an Excel spreadsheet without identifiers. Z-tests were preformed to determine statistical significance between variables with the exception of age where Student t-test was used. A p-value $\leq 0.05$ was considered significant. Initial data analysis was completed by one of the authors and reviewed by all authors to ensure agreement of observed trends and conclusions.

\section{Ethics}

Ethics approval for this study was obtained from the University of Ottawa Research Ethics Board (H-11-21-7333). In addition, we completed the ethics assessment tool from $\mathrm{PHO}$ which generated a score of zero, indicating no risk. Data pertaining to case numbers and distributions by gender were compiled based on publicly reported information from the Ontario Ministry of Health. Additional information on demographics and risk factors for syphilis infection did not include identifiable information. Data collection did not involve direct contact with cases.

\section{Results}

\section{Epidemiology review of syphilis cases: 2010-2019}

In Ottawa, from 2010-2019, 1,693 syphilis diagnoses were reported; 878 were infectious (primary, secondary, or early latent) and 815 were late latent (Table 1). For infectious syphilis, the annual number of reported cases remained relatively stable in the first half of our review period (2010-2014). During this time, 206 cases were reported, averaging approximately 41 cases per year (range $30-50$ ); $96 \%$ of these cases were male and $4 \%$ were female. Interestingly, beginning in 2015, infectious syphilis cases began to surge increasing to 100 with incremental increases observed each year onward (Figure 1). In the latter half of the decade, infectious syphilis rates more than tripled, yielding 672 cases over these five years with 171 cases in 2019 alone (change from 10,7/100,000 persons in 2016 to $15,6 / 100,000$ persons in 2019).

Though most diagnoses of infectious syphilis in Ottawa continue to involve gbMSM, the proportion of cases reported among this group decreased from $91 \%$ to $73 \%$ over the 10 -year period. The proportion of cases diagnosed among HIV-positive persons also declined over the same period with $53 \%$ of syphilis cases 
Table 1: Yearly count of syphilis diagnoses by stage

\begin{tabular}{|l|r|r|}
\hline \multicolumn{1}{|c|}{ Year } & Infectious syphilis (\#) & Late latent syphilis (\#) \\
\hline 2010 & 50 & 126 \\
\hline 2011 & 46 & 104 \\
\hline 2012 & 37 & 93 \\
\hline 2013 & 30 & 77 \\
\hline 2014 & 43 & 80 \\
\hline 2015 & 100 & 65 \\
\hline 2016 & 135 & 79 \\
\hline 2017 & 106 & 63 \\
\hline 2018 & 160 & 71 \\
\hline 2019 & 171 & 57 \\
\hline Total & 878 & 815 \\
\hline
\end{tabular}

Figure 1: Infectious syphilis diagnoses

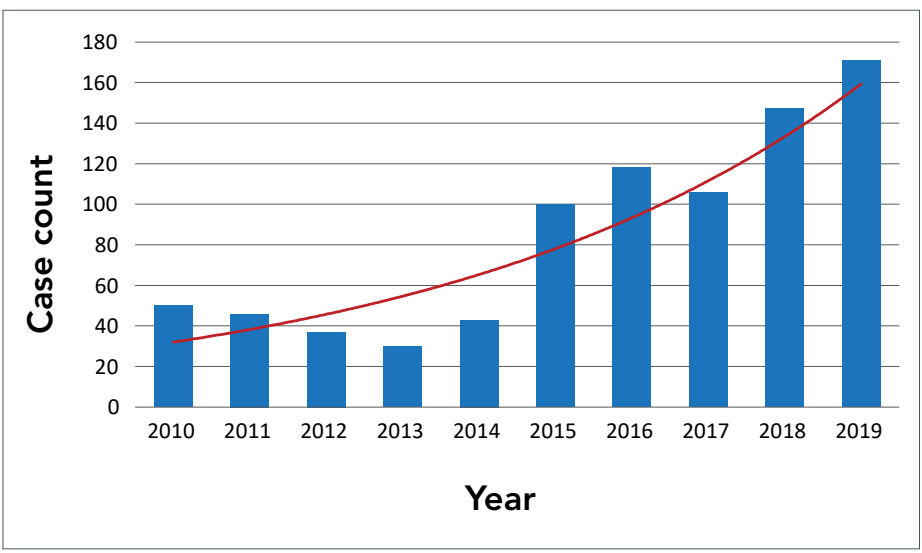

Note: The red line is the exponential trendline for infectious syphilis rates

in 2010 reported among persons living with HIV compared to $19 \%$ in 2019 . The nearly $20 \%$ decrease in syphilis cases among gbMSM has corresponded with an increase in the number of syphilis cases reported among heterosexual men and women.

In terms of late latent diagnoses in 2010-2019, rates varied by year. Inversely to the rates of infectious syphilis which increased over the 10-year period, the rates of late latent syphilis decreased from 126 diagnoses in 2010 to 57 in 2019 (Figure 2).

Diagnoses of late latent syphilis were relatively similar between males and females and most commonly reported among individuals with opposite sex sexual partners, who immigrated from a syphilis endemic region (identified during immigration medical examination), and in those with new onset memory loss (identified during dementia workup).

\section{In-depth review of infectious syphilis cases: 2015-2019}

While the incidence of infectious syphilis remained relatively stable from 2010-2014 at an average 41 cases per year, new diagnoses doubled to 100 in 2015 and nearly doubled again to
Figure 2: Late latent syphilis diagnoses

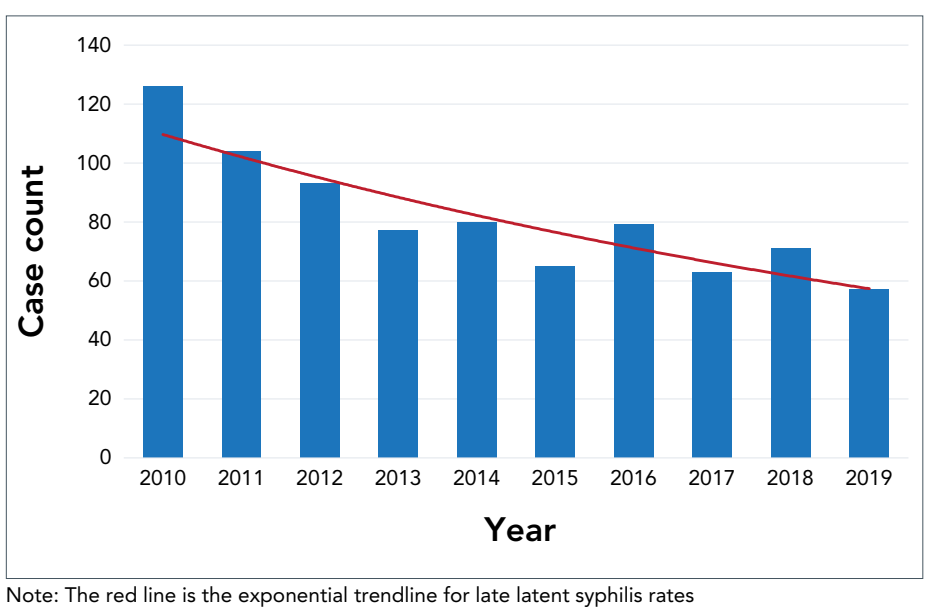

171 in 2019. Since 2015 marked a point of substantial increase in syphilis diagnoses, a more detailed chart review was completed for cases from 2015 to 2019. Of the total 672 cases of infectious syphilis in Ottawa reported during this period by the Ontario Ministry of Health, 637 charts (95\%) were available for review (Table 2).

Table 2: Demographics of cases of infectious syphilis in Ottawa

\begin{tabular}{|l|r|r|r|r|r|}
\hline Characteristic & 2015 & \multicolumn{1}{|c|}{2016} & \multicolumn{1}{|c|}{2017} & \multicolumn{1}{|c|}{2018} & 2019 \\
\hline Number of cases & 100 & 118 & 106 & 147 & 166 \\
\hline Male & $98 \%$ & $95 \%$ & $93 \%$ & $97 \%$ & $90 \%$ \\
\hline Female & $2 \%$ & $5 \%$ & $7 \%$ & $3 \%$ & $10 \%$ \\
\hline $\begin{array}{l}\text { Average age } \\
\text { (years) }\end{array}$ & 46.8 & 41.1 & 38.9 & 38.0 & 39.6 \\
\hline MSM & $46 \%$ & $84 \%$ & $87 \%$ & $91 \%$ & $73 \%$ \\
\hline MSW & $8 \%$ & $7 \%$ & $4 \%$ & $4 \%$ & $13 \%$ \\
\hline WSM & $64 \%$ & $64 \%$ & $72 \%$ & $65 \%$ & $60 \%$ \\
\hline Bisexual & $38 \%$ & $36 \%$ & $36 \%$ & $30 \%$ & $20 \%$ \\
\hline Previous STI & $4 \%$ & $4 \%$ & $2 \%$ & $4 \%$ \\
\hline HIV+ & $\begin{array}{l}\text { Abbreviations: HIV+, human immunodeficiency virus-positive; MSM, men who have sex with men; } \\
\text { MSW, men who have sex with women; STI, sexually transmitted infection; WSM, women who } \\
\text { have sex with men }\end{array}$ & & & $3 \%$ & $10 \%$ \\
\hline
\end{tabular}

Interestingly, the gender distribution and proportion of gbMSM accounting for new diagnoses remained stable during the initial surge from 2015 to 2018 but shifted in 2019. Similar to the period from 2010-2014, despite the increase in number, men still accounted for an average of $96 \%$ of cases of infectious syphilis from 2015-2018, while women made up about $4 \%$ of cases. In 2019, however, $10 \%$ of new diagnoses were among women $(p=0.006)$. Further, while the proportion of new diagnoses 
among women increased overall 2.5-fold, the absolute number increased $850 \%$. Similarly, whereas heterosexual men made up, on average, $6 \%$ of cases of infectious syphilis from 2015-2018, they accounted for $13 \%$ in 2019 ( $p=0.0005)$, representing a $550 \%$ increase in absolute number. Taken together, the proportion of new diagnoses of infectious syphilis among heterosexual men and women in Ottawa increased from 5\% in 2015 to $23 \%$ in 2019 ( $p=0.0003$ ). Overall, the male-to-female ratio within this group remained relatively stable across the five years. With the increase in diagnoses among heterosexuals, gbMSM accounted for a lower proportion of new diagnoses in 2019; dropping from $86 \%$ in 2015 to $73 \%$ in 2019 , although this difference did not reach statistical significance $(p=0.24)$. The absolute number of cases of infectious syphilis among gbMSM still increased $41 \%$ from 86 cases in 2015 to 133 cases in 2018 and 121 in 2019. Interestingly, diagnoses among individuals who self-identified as bisexual remained low and stable in both proportion and number across the five years.

For risk factors, a previous diagnosis of a sexually transmitted infection (STI) was present in $60 \%-70 \%$ of cases of infectious syphilis in Ottawa in 2015-2019. Notably, the proportion of those with a prior STI was relatively stable over the five years. In contrast, the proportion of new syphilis cases with a prior or concurrent diagnosis of HIV infection, all among gbMSM, declined from $36 \%-38 \%$ in $2015-2017$ to $20 \%$ in 2019 ( $p=0.002$ ). This decrease is not solely accounted for by the shift of syphilis into the predominantly HIV-negative heterosexual population. Among gbMSM in Ottawa with a diagnosis of infectious syphilis, the proportion who were HIV-negative increased from $58 \%$ in 2015-2017 to 73\% in 2019 ( $p=0.082)$ although this shift did not quite reach statistical significance.

Finally, while the distribution in gender and sexual orientation among new syphilis diagnoses in Ottawa appears to have shifted late in the five years under study, the average age at diagnosis decreased somewhat from 46.8 years in 2015 to 41.1 years in 2016 and 38.0-39.6 years in 2017-2019 $(p=0.094)$.

\section{Review of antenatal syphilis diagnoses}

Considering the increasing number of syphilis cases among women of childbearing age and risk of perinatal transmission from untreated syphilis in pregnancy, we reviewed all positive syphilis tests found on antenatal screening in Ottawa from January 2015 to July 2021. Of a total of 37 positive tests among pregnant females, 17 reported cases of syphilis were identified in 15 patients. As seen in Figure 3, the number of antenatal syphilis cases in Ottawa has been steadily increasing since 2015 (from no reported cases in 2015 to four cases in mid-2021). While the 2021 data for pregnant women was incomplete at the time of this review, the exponential trendline for antenatal syphilis indicates further upward trends in diagnoses over time. Of the 17 reported cases of syphilis during the review period, three were cases of early infectious syphilis and eight were latent syphilis - as either latent syphilis of unknown duration or late latent. The average age of women who tested positive
Figure 3: Infectious syphilis diagnoses in pregnancy

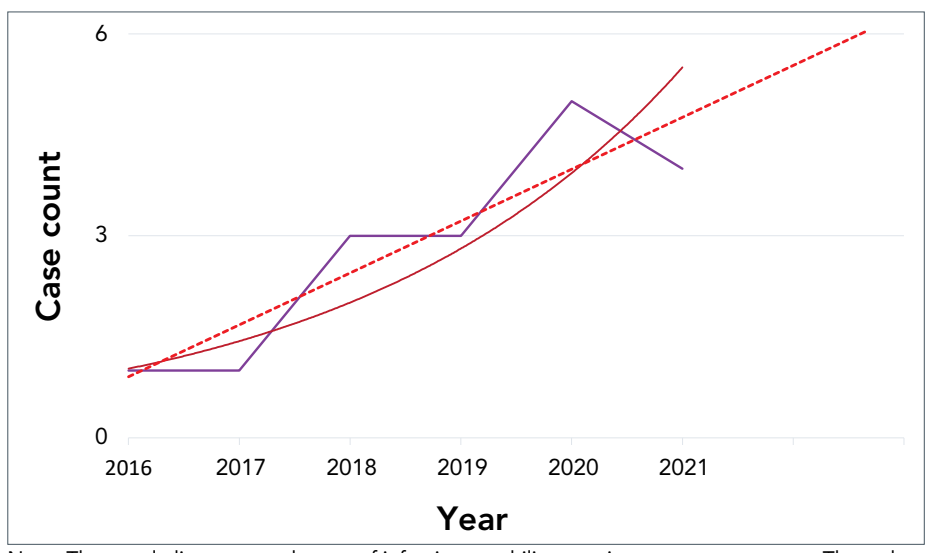

Note: The purple line reported cases of infectious syphilis cases in pregnancy per year. The red line is the exponential trendline for infectious syphilis cases in pregnancy. The dotted line is the linear forecast for future incidence of infectious syphilis in pregnancy

for syphilis during antenatal screening was 27.7 years (range: 17-39 years) and ethnicity varied. The remaining six positive tests were in pregnant women who had a reported case of syphilis in Ottawa, but reported having been previously treated for syphilis outside of Ontario (i.e. had no prior follow-up in Integrated Public Health Information System database) or had a reactive chemiluminescent microparticle immunoassay test with negative rapid plasma reagin and a negative Treponema pallidum particle agglutination test, which are laboratory results consistent with a false positive screening. Of the pregnancies that were maintained and/or completed at the time of this review, none was diagnosed with congenital syphilis based on serologic testing and physical examination of the infant. All infants were referred to the care of paediatric infectious diseases specialists for monitoring. To date, there have been no reported diagnoses of congenital syphilis in Ottawa.

\section{Discussion}

Our review of syphilis diagnoses in Ottawa from 2010-2019 showed a decrease in non-infectious (late latent syphilis) and a marked increase in the incidence of infectious syphilis over the study period and a shift in demographic distribution. Overall, the mean age at diagnosis decreased slightly with an increase in cases among heterosexual men and women and among HIV-negative gbMSM. The shift in syphilis toward heterosexual populations, particularly women, has been similarly observed across Canada with Public Health Agency of Canada (PHAC) reporting a $740 \%$ increase in infectious syphilis in this population from 2016-2020 (5), with the highest rates reported among women of childbearing age. While gbMSM still account for the vast majority of syphilis diagnoses, the proportion of cases in this population has decreased in tandem with increases among heterosexuals $(4,5)$. Similar upwards trends are now observed among women during pregnancy, with forecasted increases anticipated; however, no congenital syphilis diagnoses have been observed in Ottawa to date. 


\section{Considerations for clinical and public health practices}

First, the increasing rates of infectious syphilis among women, particularly those of childbearing age, pose a concern for possible future increases in congenital syphilis. Canada-wide, $\mathrm{PHAC}$ has already reported a significant increase in the number of confirmed congenital syphilis cases from four in 2016 to 73 in 2020 (5). PHAC noted that this increase is proportional to the increase in infectious syphilis diagnoses among women aged 15-24 years (5). While we have yet to document any confirmed or suspected cases of congenital syphilis in Ottawa, the changing landscape in our region augurs poorly that this will remain the case. As seen in Figure 3, the linear forecast for infectious syphilis among pregnant women in Ottawa projects continued future increases in this group. In view of this, clinicians and public health practitioners should consider more consistent screening of women of childbearing age for syphilis to avoid poor health outcomes in this group and prevent possible cases of congenital syphilis (9-11). Both increased awareness and robust clinical guidance are recommended to assist practitioners in screening for, treating and monitoring syphilis in pregnant women, including screening early in pregnancy and repeat testing in the third trimester if ongoing risk is identified (12). Locally, our public health unit provides comprehensive follow-up for all positive syphilis tests found on antenatal screening. This includes referrals to appropriate specialty care and follow-up syphilis serology during pregnancy and for the infant in the first year of life.

A second point of interest is the increasing proportion of new syphilis diagnoses in Ottawa among HIV-negative gbMSM. Commonly, the increasing incidence of syphilis in this group is attributed to the uptake of HIV preexposure prophylaxis (PrEP) and a concurrent decrease in condom use $(13,14)$. A cause and effect here, however, is very unlikely (15). First, the surge in infectious syphilis in Ottawa first began in 2015. Accessed by some individuals through a single clinic in Ottawa began on November 2015. PrEP was not approved by Health Canada until 2016 (16) and has only become more generally available in recent years. Second, when we examined the rates of STIs, including syphilis, among gbMSM using PrEP in Ottawa in 2018, we did not find a higher incidence among men using PrEP compared to gbMSM in the general population (17). Thus, uptake of PrEP is unlikely to be the cause of the increase in infectious syphilis among HIV-negative gbMSM. In view of this, the vulnerability for HIV infection documented among individuals who acquire syphilis is of enhanced concern. The United States' Centers for Disease Control and Prevention estimates that one in $18 \mathrm{gbMSM}$ are infected with HIV within one year of acquiring syphilis (18). This risk is amplified in racialized populations with the Centers for Disease Control and Prevention estimating the lifetime risk of HIV infection to be one in two for African, Caribbean or Black gbMSM, and one in four for Latino or Hispanic gbMSM (18). Given the enhanced risk of HIV infection among gbMSM diagnosed with syphilis, we suggest HIV PrEP be offered to persons in this group. Specifically, for any gbMSM diagnosed with primary, secondary or early latent syphilis, we recommend an active offer of HIV PrEP be made $(15,19)$. In Ottawa, this is done routinely by public health staff during follow-up and contact tracing $(15,19)$. Since doing so, we have seen a decline in HIV infections in Ottawa (20) though this observation is confounded by increasing uptake of PrEP through several clinics and family physicians across the city and by decreased HIV testing due to the coronavirus disease 2019 pandemic in 2020.

Considering the changes in syphilis epidemiology observed in Ottawa and elsewhere in Canada and the increasing proportion of new HIV diagnoses now occurring in heterosexual men and women locally, offers of PrEP should likely be expanded to other groups beyond gbMSM (21). To mitigate ongoing HIV transmission in heterosexual groups, we suggest applying some of the same criteria used for PrEP in gbMSM (19); 1) for all heterosexual groups with a repeat diagnosis of STI (including syphilis) and 2) women with a diagnosis of infectious syphilis, gonorrhea or hepatis $C(21,22)$. While no heterosexual men or women in our review had a previous or concurrent diagnosis of HIV at the time of syphilis diagnosis, persons with opposite sex partners accounted for $9 \%(n=80 / 878)$ of all infectious syphilis diagnoses and $5 \%(n=21 / 413)$ of all patients with repeat STIs, which can potentially elevate HIV acquisition risk $(21,22)$. Offering PrEP to these individuals, in addition to gbMSM, could be a useful strategy to improve HIV prevention efforts.

Our third point relates to the decrease in non-infectious-or late latent-syphilis diagnoses observed over the 10-year review period. Though the exact reasons for this decline in late latent syphilis cases is not certain, it could relate to 1) increased awareness, testing for and treatment of infectious syphilis over the past 20 years resulting in fewer cases becoming late latent, and/or 2) changes in case classification (12), particularly those classified as latent syphilis of unknown duration. While this latter classification supports longer treatment in individuals where syphilis may or may not have been recently acquired, it muddies surveillance data.

Lastly, based on our data, we suggest broader public awareness campaigns and increased screening among heterosexuals, and reinstatement of syphilis of undetermined duration as a reportable stage of infection (22). Given the known health complications of syphilis, it is concerning that despite the documented shift of new cases into the heterosexual population, screening efforts continue to target almost exclusively gbMSM. Social media campaigns and other strategies should be employed to ensure heterosexual men and women are aware of syphilis and the benefits of testing. From a public health perspective, we also advocate for the inclusion of syphilis of undetermined duration in surveillance data. Presently, these cases are typically captured as late latent though it is unknown if syphilis was recently acquired (23). Classifying syphilis of undetermined duration as late latent means these cases will not be included in the changing epidemiology and will bias surveillance away from non-gbMSM populations where routine 
testing is less common. Also, public health follow-up and contact tracing will not occur in these cases, allowing the potential for onward transmission $(12,23,24)$.

\section{Limitations}

Our study has several limitations. The data reported in this review are based on information collected and input by public health nurses during routine syphilis follow-up. It is possible that the consistency of the data was subject to some variation. Further, the breadth of information related to infectious syphilis is limited to information specified in the public health reporting system, which does not capture data on transgender, ethnicity and some infection-specific risk factors (e.g. sex with bisexual partners, group sex, etc.). There were some differences between the number of cases reported by $\mathrm{PHO}$ and the number reported by $\mathrm{OPH}$. This likely relates to changes in diagnosis, staging, or responsible public health unit not adjusted for by PHO. Finally, in this review, we do not report on cases diagnosed as syphilis of unknown duration as this is no longer a reportable stage. It is possible that the distribution of cases of infectious syphilis, particularly among heterosexual groups, is higher than what is reported.

\section{Conclusion}

Findings from our review of syphilis epidemiology in Ottawa support our clinical suspicions and align with recent reports from other Canadian cities and provinces. While the incidence of infectious syphilis remained relatively stable in the first half of our review period (2010-2014), cases surged in the period of 2015 to 2019. Superimposed on this was the notable increase in diagnoses among individuals previously considered at low risk, specifically heterosexual men and women. While 10 years ago, heterosexuals accounted for approximately $5 \%$ of infectious syphilis cases in Ottawa, by 2019 they accounted for nearly one-quarter. Cases also increased among HIV-negative gbMSM with the proportion of cases with concurrent HIV infection decreasing from $38 \%$ to $20 \%$. Based on our findings, we recommend clinicians and public health practitioners increase syphilis screening beyond the gbMSM population and strengthen contact tracing efforts for persons who may have been exposed to syphilis. Increased efforts should also be made to strengthen HIV prevention efforts, such as PrEP, to heterosexual men and women diagnosed with infectious syphilis. In response to rising rates of infectious syphilis among heterosexual women, future research must be done to help explore syphilis risk factors within this group. We also strongly advocate that prenatal syphilis screening be done as early as possible and, where indicated, screening be repeated during pregnancy and/or near term. As the syphilis epidemic continues to evolve in Ottawa, ongoing surveillance will continue to play a crucial role. While public health resources must ensure ongoing support for populations already impacted by syphilis, they must also be flexible and address emerging trends in the epidemic.

\section{Authors' statement}

LO completed the initial data extraction of syphilis diagnoses in Ottawa from 2010-2019. All authors were involved in the data analysis, article writing, editing, submission, and approval of this article.

The content and view expressed in this article are those of the authors and do not necessarily reflect those of the Government of Canada.

\section{Competing interests}

None.

\section{Acknowledgements}

The authors would like to thank Ottawa Public Health for approving this review of syphilis epidemiology in Ottawa. In addition, LO would like to acknowledge the Canadian Institutes of Health Research for her Vanier Scholarship and POB would like to acknowledge the Ontario HIV Treatment Network for his Chair in Public Health and HIV Prevention.

\section{Funding}

None.

\section{References}

1. Totten $S$, MacLean R, Payne E. Infectious syphilis in Canada: 2003-2012. Can Commun Dis Rep 2015;41(2):30-4. DOI PubMed

2. Choudhri Y, Miller J, Sandhu J, Leon A, Aho J. Infectious and congenital syphilis in Canada, 2010-2015. Can Commun Dis Rep 2018;44(2):43-8. DOI PubMed

3. Public Health Agency of Canada. Syphilis in Canada: Technical report on epidemiological trends, determinants, and interventions. Epidemiological trends of syphilis in Canada, 2009-2018. Ottawa (ON): PHAC; 2020. https:// www.canada.ca/en/services/health/publications/diseasesconditions/syphilis-epidemiological-report.html\#3

4. Public Health Agency of Canada. Report on sexually transmitted infections in Canada, 2018. Age and sex distribution. Ottawa (ON): PHAC; 2021. https://www. canada.ca/en/public-health/services/publications/ diseases-conditions/report-sexually-transmitted-infectionscanada-2018.html\#a6.2 
5. Public Health Agency of Canada. Infectious syphilis and congenital syphilis in Canada, 2020. Ottawa (ON): PHAC; 2021. https://www.canada.ca/en/public-health/services/ publications/diseases-conditions/infectious-syphiliscongenital-syphilis-canada-2020.html

6. Ottawa Public Health. Sexually transmitted infections and sexual health in Ottawa. Ottawa (ON): OPH; 2011. https://www.ottawapublichealth.ca/en/reports-researchand-statistics/resources/Documents/sexually_transmitted_ infections_2011_en.pdf

7. Leber A, MacPherson P, Lee BC. Epidemiology of infectious syphilis in Ottawa. Recurring themes revisited. Can J Public Health 2008;99(5):401-5. DOI PubMed

8. Public Health Ontario. Infectious Disease Trends in Ontario. Syphilis: Infectious rates and cases for all ages and all sexes in Ottawa. Toronto (ON): PHO; 2021. https://www. publichealthontario.ca/en/data-and-analysis/infectiousdisease/reportable-disease-trends-annually\#/54

9. Public Health Agency of Canada. Syphilis guide: Screening and diagnostic testing. Ottawa (ON): PHAC; 2020. https://www.canada.ca/en/public-health/services/infectiousdiseases/sexual-health-sexually-transmitted-infections/ canadian-guidelines/syphilis/screening-diagnostic-testing. html

10. Singh $A E$, Levett $P N$, Fonseca K, Jayaraman GC, Lee BE. Canadian Public Health Laboratory Network laboratory guidelines for congenital syphilis and syphilis screening in pregnant women in Canada. Can J Infect Dis Med Microbiol 2015;26 Suppl A:23A-8A. DOI PubMed

11. Robinson JL. Congenital syphilis: No longer just of historical interest. Can Paed Soc 2018;14:337. https://cps.ca/en/ documents/position/congenital-syphilis

12. Public Health Agency of Canada. Syphilis guide: Treatment and follow-up. Ottawa (ON): PHAC; 2020. https://www. canada.ca/en/public-health/services/infectious-diseases/ sexual-health-sexually-transmitted-infections/canadianguidelines/syphilis/treatment-follow-up.html

13. Grov C, Whitfield TH, Rendina HJ, Ventuneac A, Parsons JT. Willingness to take PrEP and potential risk compensation among highly sexually active gay and bisexual men. AIDS Behav 2015;19(12):2234-44. DOl PubMed

14. Harawa NT, Holloway IW, Leibowitz A, Weiss R, Gildner J, Landovitz RJ, Perez MJ, Kulkarni S, Rotheram-Borus MJ, Shoptaw S. Serious concerns regarding a meta-analysis of preexposure prophylaxis use and STI acquisition. AIDS 2017;31(5):739-40. DOl PubMed
15. Orser $L, O^{\prime}$ Byrne $P$. The role of public health units in the delivery of HIV pre-exposure prophylaxis (PrEP). Can J Public Health 2019;110(1):72-5. DOI PubMed

16. Health Canada. Notice of Compliance information: Truvada. Ottawa (ON): HC; 2016. https://health-products.canada.ca/ noc-ac/info.do?lang $=e n \&$ no $=17808$

17. Razmjou SM, Charest M, O'Byrne $P$, MacPherson $P$. Pre-Exposure Prophylaxis Among Gay, Bisexual, and Other Men Who Have Sex With Men in Ottawa: A Real World View With Benefits Beyond HIV Risk Reduction. Int J Sex Heal 2021. DOI

18. Centers for Disease Control and Prevention (CDC). Sexually Transmitted Infections Treatment Guidelines, 2021. Atlanta (GA): CDC; 2021. https://www.cdc.gov/std/ treatment-guidelines/toc.htm

19. O'Byrne P, MacPherson P, Orser L, Jacob JD, Holmes D. PrEP-RN: Clinical Considerations and Protocols for Nurse-Led PrEP. J Assoc Nurses AIDS Care 2019;30(3):301-11. DOI PubMed

20. Public Health Ontario. Infectious Disease Trends in Ontario. HIV: Infectious rates and cases for all ages, for all sexes in Ottawa. Toronto (ON): PHO; 2021. https://www. publichealthontario.ca/en/data-and-analysis/infectiousdisease/reportable-disease-trends-annually\#/28

21. Orser $L, O^{\prime}$ Byrne P. Examining patient characteristics and HIV-related risks among women with syphilis as indicators for pre-exposure prophylaxis in a nurse-led program (PrEP-RN). Int J STD AIDS 2021;32(1):45-51. DOI PubMed

22. Centers for Disease Control and Prevention. Preexposure prophylaxis for the prevention of HIV infection in the United States - 2021 update. CDC; 2021. https://www.cdc.gov/hiv/ pdf/risk/prep/cdc-hiv-prep-guidelines-2021.pdf

23. O'Byrne $P$, Orser L, MacPherson P. Discussing current syphilis case definitions: A proposal for a "probable infectious" case. Public Health Nurs 2021;38(3):390-5. DOI PubMed

24. Ministry of Health and Long-term Care; Provincial Infectious Diseases Advisory Committee. Sexually Transmitted Infections Case Management and Contact Tracing Best Practice Recommendations. Toronto (ON): MHLTC; 2009. https://www.publichealthontario.ca/-/media/ documents/S/2009/sti-case-management-contact-tracing. pdf 